Results Thirty VLBW children were assessed at mean age of 7.5 years; 30 children born at term (matched for age, sex, and family income) served as controls. WISC-III scores were comparable between the two groups $(99.8 \pm 2.4$ and $105.8 \pm 1.7 ; \mathrm{p}=$ 0.072 in VLBW and control group, respectively) as well as the WRAT scores. GMFCS-mild dysfunction was found only in 2 children (6.7\%) of VLBWgroup. In contrast, VLBW children had more behavioural/emotional problems, especially in attention deficit/hyperactive $(26.7 \%$ in VLBW group vs.3.3\% in controls, $\mathrm{p}=0.026)$.

Conclusions In our cohort study, VLBW children at school age are at higher risk for behavioural/emotional problems, especially in attention deficit compared with children born at term. However, no differences in cognitive, academic, and gross motor function were found.

\section{PO-0643 MACROPHAGE ACTIVATION SYNDROME IN A NEWBORN INFANT BORN TO A MOTHER WITH AUTOIMMUNE DISEASE}

JH Park, SH Kim, DC Jeong, SY Kim. Pediatrics, The Catholic University of Korea College of Medicine, Seoul, Korea

\subsection{6/archdischild-2014-307384.1284}

We reported a newborn infant with macrophage activation syndrome (MAS) born from a mother with positive anti-nuclear (ANA) and anti-SSA/Ro antibodies. The 2,500 g girl was born at $37^{+6}$ weeks of gestation in good condition. Mother had been diagnosed with adult onset Still disease 10 years previously. During pregnancy, she did not take any medication as she was free of symptoms. The baby was admitted due to tachypnea and fever $12 \mathrm{~h}$ after birth. Initial laboratory findings showed mild anaemia with thrombocytopenia, and mild elevation of alanine aminotransferase (ALT). A work-up of infectious aetiology, including agents responsible for congenital infection, was negative. On the 10th hospital day (HD), the baby showed severe abdominal distension caused by hepatosplenomegaly, and persistent, high fever despite empirical antibiotic therapy. We identified positive ANA and anti-SSA/Ro antibodies from the infant, compatible with those found in the mother. The baby's electrocardiography was normal. On the 18th HD, she showed deterioration of overall condition with high ferritin, ALT, and profound

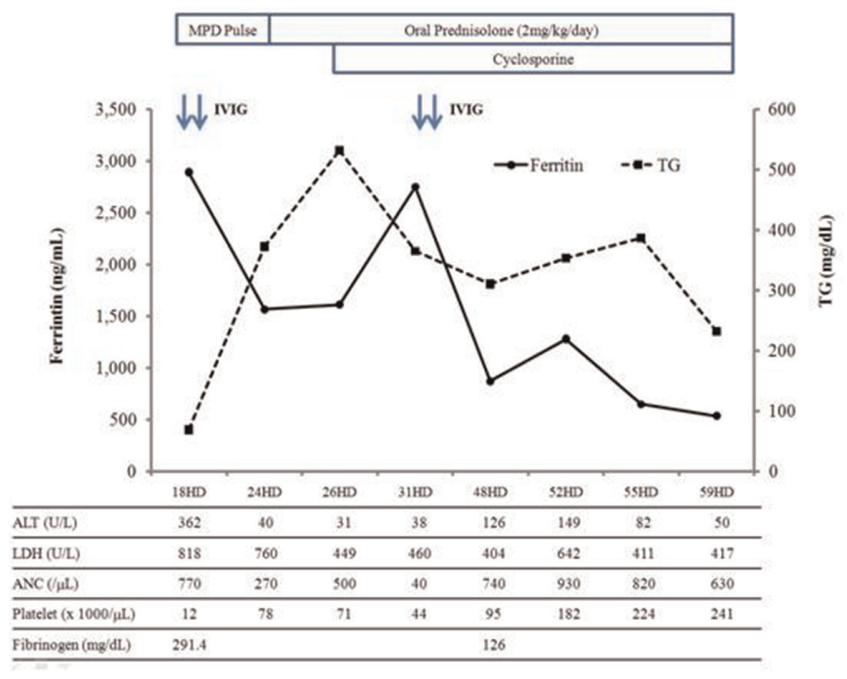

Abstract P0-0643 Figure 1 thrombocytopenia. The baby received intravenous immunoglobulin, steroid (pulse and oral), and cyclosporine. Gene study for perforin, K-ras, and N-ras was negative. Her general condition showed improvement after treatment, although mild fever and organomegaly remained. We maintained high dose steroid and cyclosporine, and all medication was tapered and stopped at 12 weeks of age. We suggest that transplacental transfer of maternal auto-antibodies may be associated with the infant's MAS.

\section{P0-0644 WHITE LIGHT SPECTROSCOPIC TRANSCUTANEOUS MEASUREMENTS OF BILIRUBIN LEVELS IN JAUNDICED INFANTS INCLUDING KRAMER ZONES}

${ }^{1} \mathrm{H}$ Rabe, ${ }^{2} \mathrm{M}$ Olbert, ${ }^{3} \mathrm{~S}$ SobowiecKouman, ${ }^{3} \mathrm{R}$ Fernandez, ${ }^{3} \mathrm{P}$ Amess, ${ }^{4} \mathrm{H}$ Jungmann. ${ }^{1}$ Neonatology, Royal Sussex County Hospital, Brighton, UK; ${ }^{2}$ Research and Development, MBR Optical Systems, Wuppertal, Germany; ${ }^{3}$ Neonatology, Brighton and Sussex University Hospitals, Brighton, UK; ${ }^{4}$ Research and Development, MBR OpticalSystems, Wuppertal, Germany

\subsection{6/archdischild-2014-307384.1285}

Background Jaundice is a common problem affecting $8-10 \%$ of preterm and term newborn babies in the first week of life. Efforts have been made to have non-invasive diagnostic devices available for reliable and quick bedside testing of bilirubin levels in order to avoid painful blood taking and delays due to awaiting results from the laboratory. Several transcutaneous devices can estimate bilirubin levels in the newborn and have mostly been tested in term infants. The correlation co-efficient between transcutaneous and laboratory values have been reported to be 0.46 to 0.89 depending on the device. The device cannot be used after phototherapy has started as bilirubin isomeres are produced. Thus a non-invasive device which can measure after the start of phototherapy in preterm and term infants is warranted.

Objective To establish whether the non-invasive white light spectroscopic (WLS) device (Bilispect (R), MBR Optical Systems, Wuppertal, Germany) can measure bilirubin in the skin of preterm and term jaundiced babies before and after phototherapy.

Methods Prospective single centre study of preterm and term infants who had their bilirubin levels taken for clinical reason in a convenience cohort sample. Best measurement site on skin was determined by comparing WLS measurements at 4 Kramer zones (forehead, sternum, forearm and foot) in 9 infants. Forearm fared best. Prospective WLS measurements were taken within 15 min of the blood test at the forearm of the baby. Statistical comparison was performed by Bland-Altman method. Intra- and inter-observer variability were calculated by using repeated measurements and two observers. The ethics committee issued a favourable opinion and informed consent was obtained from parents.

Results Comparisons were obtained in 47 preterm and term infants (range 24-40 weeks gestation, birth weight 564-4220 g), who had 51 paired bilirubin samples done. Ten were taken after phototherapy had been started. Correlation coefficients for samples taken after phototherapy was 0.87 and 0.9 without phototherapy. Intra-observer and inter-observer variability were 0.67 and 0.69 respectively.

Conclusions The results show a good correlation between the laboratory and non-invasive bilirubin values. The WLS seems to be a suitable method for estimating bilirubin levels after phototherapy has started and particularly in preterm infants. Further field studies re required in order to obtain nomograms for this device. 